\title{
UNIT FRACTIONS AND THE ERDÖS-STRAUS CONJECTURE
}

\author{
A.G. Shannon \\ Emeritus Professor, University of Technology Sydney, NSW 2007, \\ \& \\ Campion College, PO Box 3052, Toongabbie East, NSW 2146, Australia \\ J.V. Leyendekkers \\ Faculty of Science, The University of Sydney, NSW 2006, Australia \\ t.shannon@campion.edu.au, tshannon38@gmail.com
}

\begin{abstract}
This note considers some aspects of finite sums of unit fractions, including associated recurrence relations and conjectures in the context of experimental mathematics. Unit fractions provide a unifying theme.
\end{abstract}

\section{Indexing terms/Keywords}

Arithmetic; geometric and harmonic means; ceiling and floor functions; conjectures; continued fractions; Egyptian fractions,;Fibonacci numbers; greedy algorithms; symmetric functions; unit fractions.

\section{Academic Discipline And Sub-Disciplines}

Number Theory, History; Education;

\section{SUBJECT CLASSIFICATION}

AMS Classification Numbers: 11A41, 11-01.

\section{TYPE (METHOD/APPROACH)}

This paper considers conjectures in general and their mathematical context with particular applications to the Erdös-Straus conjecture with unit fractions as the unifying theme in the context of experimental mathematics.

\section{INTRODUCTION}

As an exercise in experimental mathematics [2], this note aims to elaborate some conjectures related to Egyptian fractions and harmonic numbers and to explore them with some recurrence relations and continued fractions. In the context of teaching and learning in general they implicitly involve the relatively neglected educational concepts of functional literacy and numeracy [27].

Conjectures have an inherent fascination and challenge because we can neither prove them nor find counter examples [10]. They can encourage non-standard mathematical skills such as shrewd guessing (or conjecturing) [21], considering integer structure [16], and new approaches to viewing the Cartesian plane [8] in the context of the history of mathematical conjectures [9].

\section{EGYPTIAN FRACTIONS}

Egyptian fractions are finite sums of distinct unit fractions. Unit fractions are rational numbers with unit numerators and positive integers as denominators. For example, $1 / 2+1 / 3+1 / 6$ qualifies as an Egyptian fraction. We shall also consider briefly such non-standard properties as repeated fractions or negative integers [23].

Every positive rational number can be represented by an Egyptian fraction, so that this was one way the ancient Egyptians were able to use these sums as notation for rational numbers [12]. For instance,

which can be represented more generally by

$$
\frac{2}{21}=\frac{1}{14}+\frac{1}{42}
$$

$$
\frac{2}{p q}=\frac{1}{\left(\frac{p+1}{2}\right) q}+\frac{1}{\left(\frac{p+1}{2}\right) p q} .
$$

Fibonacci's Liber Abaci used what we know as vulgar fractions to replace Egyptian fractions [24]. For example,

$$
\frac{8}{11}=\frac{6}{11}+\frac{2}{11}=\left(\frac{1}{2}+\frac{1}{22}\right)+\left(\frac{1}{6}+\frac{1}{66}\right)
$$

in which the numerator is split as follows

$$
\frac{a}{b}=\frac{p+q}{b}
$$




$$
p|b+1, q| b=1 \text {. }
$$

Fibonacci went further by suggesting what is now referred to as a 'greedy algorithm' for calculating Egyptian fractions [11]. A greedy algorithm seeks locally optimal solutions to a problem at each stage of a problem solving heuristic [6]. In the example, the iterations terminate with finite a expression starting with

$$
\frac{a}{b}=\frac{1}{\lceil b / a\rceil}+\frac{(-b)(\bmod a)}{b\lceil b / a\rceil}
$$

in which $\lceil\bullet]$ represents the ceiling function. For instance, $6 / 17=1 / 3+1 / 51$.

The Erdös-Straus conjecture [1] is a related unsolved problem. It can be stated as: there exist positive integers $x, y$ and $z$ such that

$$
\frac{4}{n}=\frac{1}{x}+\frac{1}{y}+\frac{1}{z}
$$

for every integer $n \geq 2$ [3]. For example, for $n=2,(x, y, z)=(2,2,1)$; when $n=3,(x, y, z)=(2,2,3)$. When $x, y, z$ are distinct the solution represents an Egyptian fraction for 4/n [25]. Sometimes there are multiple solutions such as when $n=5,(x, y, z)=$ $(2,4,20)$ and $(2,5,10)$. This case makes us wonder if there are patterns, and there are for some $n$, but not for all $n[14]$. As with other famous conjectures, computational mathematicians have verified the truth of the conjecture for very large values of $n$, but it has not been proved for all $n$ [cf. 15].

Because of the symmetry of the right hand side of (2.1) it can be rearranged as

$$
n=\left(\frac{4}{1+B}\right) x
$$

in which $B=x(y+z) / y z$ is constant for a given $(n, x)$ pair (Table 1$)$ and corresponding $(x, y, z)$ triplets are shown in Table 2 . Thus for $n$ equal to a prime $p$

$$
p B=4 x-p
$$

and

$$
x=\frac{p+p B}{4} .
$$

$p B$ is odd and changes by 4 when $x$ changes by 1 (Table 1 ), but some $p B$ values do not give integer solutions.

$$
\text { Table 1: } p B\left[p \in \overline{3}_{4} \Rightarrow p B \in \overline{1}_{4} ; p \in \overline{1}_{4} \Rightarrow p B \in \overline{3}_{4}\right]
$$

\begin{tabular}{|c|c|c|c|c|c|c|c|c|c|c|c|c|}
\hline $\boldsymbol{p} \backslash \boldsymbol{x}$ & $\mathbf{1}$ & $\mathbf{2}$ & $\mathbf{3}$ & $\mathbf{4}$ & $\mathbf{5}$ & $\mathbf{6}$ & $\mathbf{7}$ & $\mathbf{8}$ & $\mathbf{9}$ & $\mathbf{1 0}$ & $\mathbf{1 1}$ & $\mathbf{1 2}$ \\
\hline 3 & 1 & 5 & & & & & & & & & & \\
\hline 5 & & 3 & 7 & & & & & & & & & \\
\hline 7 & & 1 & 5 & 9 & & & & & & & & \\
\hline 11 & & & 1 & 5 & & 13 & & & & & & \\
\hline 13 & & & & 3 & 7 & & & & & & & \\
\hline 17 & & & & & 3 & 7 & & & & & & \\
\hline 19 & & & & & 1 & 5 & & 13 & & 21 & & \\
\hline 23 & & & & & & 1 & 5 & 9 & 13 & 17 & & 25 \\
\hline 29 & & & & & & & & 3 & & 11 & 15 & \\
\hline 41 & & & & & & & & & & & 3 & 7 \\
\hline
\end{tabular}

Different $p$ values may have the same $p B$ values (Figure 1). In this case the primes are in the same class. For example, a $p B$ of 1 and 5 (in $\overline{1}_{4}$ ) occurs for $p=3,7,19,23, \ldots$ where $p \in \overline{3}_{4} \subset Z_{4}, p=4 r_{3}+3$ ) [17], whereas a $p B$ of 3 and 7 occurs for $\left.p \in \overline{1}_{4} \subset Z_{4}, p=4 r_{1}+1\right)$.

Figure 1: Representation of Table 5 


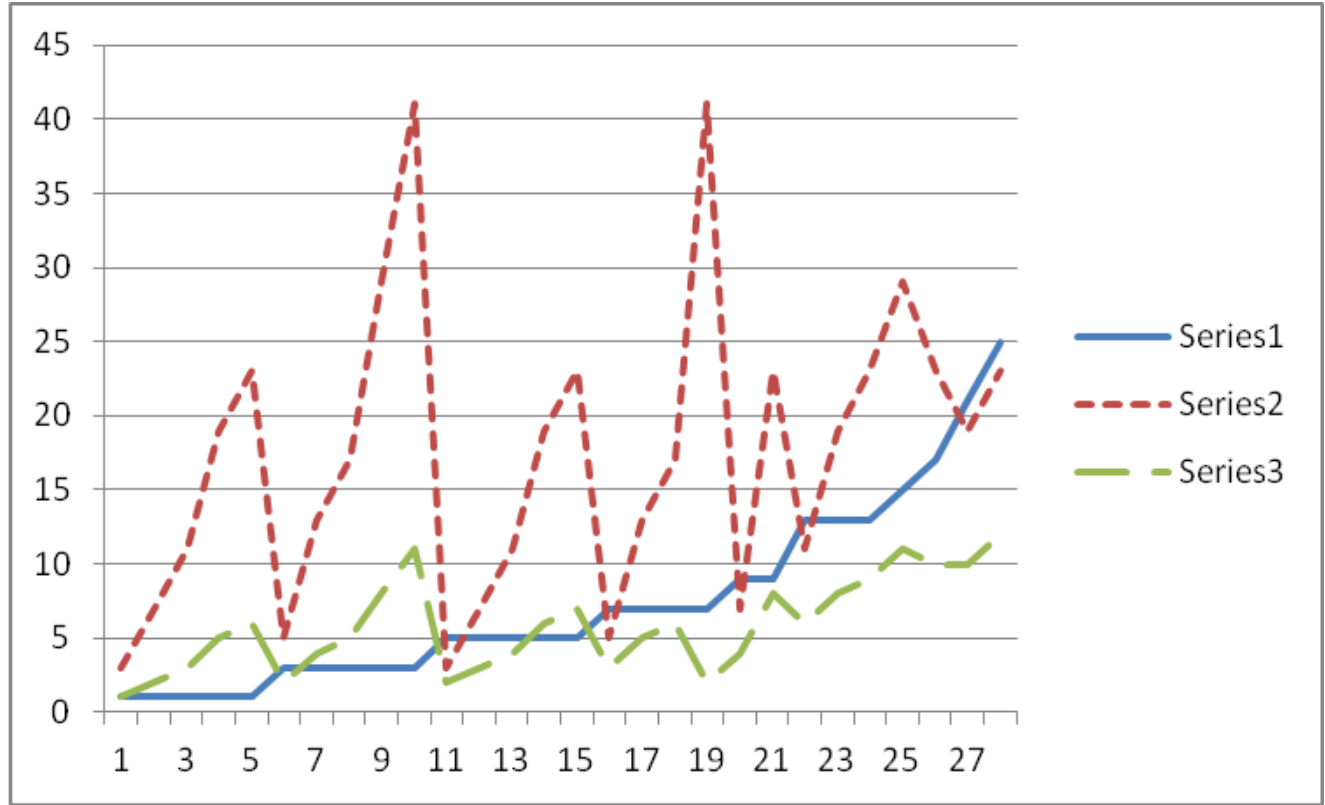

In Figure $1: x$-axis: $1-5 p B=1 ; 6-10 p B=3 ; 11-15 p B=5 ; 16-19 p B=7$;

$20-21 p B=9 ; 22-23 p B=13 ; 24 p B=15 ; 25 p B=17 ; 26 p B=21 ; 27 p B=25$.

Series 1: $p B$; Series 2: corresponding $p$; Series 3: $x$ (see Table 5).

Table 2: Equations (2.3) and (2.4)

\begin{tabular}{|c|c|c|c|c|c|c|c|c|c|}
\hline$p$ & $p B$ & $x$ & $y$ & $z$ & $p$ & $p B$ & $x$ & $y$ & $z$ \\
\hline \multirow[t]{3}{*}{3} & 1 & 1 & 4 & 12 & \multirow[t]{10}{*}{$\frac{r}{19}$} & 1 & 5 & 96 & 9120 \\
\hline & 1 & 1 & 6 & 6 & & 1 & 5 & 100 & 1900 \\
\hline & 5 & 2 & 2 & 3 & & 1 & 5 & 114 & 570 \\
\hline \multirow[t]{2}{*}{5} & 3 & 2 & 4 & 20 & & 1 & 5 & 120 & 456 \\
\hline & 3 & 2 & 5 & 10 & & 1 & 5 & 190 & 190 \\
\hline \multirow[t]{7}{*}{7} & 1 & 2 & 15 & 210 & & 5 & 6 & 23 & 2622 \\
\hline & 1 & 2 & 16 & 112 & & 5 & 6 & 30 & 95 \\
\hline & 1 & 2 & 18 & 63 & & 5 & 6 & 38 & 57 \\
\hline & 1 & 2 & 21 & 42 & & 13 & 8 & 12 & 456 \\
\hline & 1 & 2 & 28 & 28 & & 21 & 10 & 10 & 95 \\
\hline & 5 & 3 & 6 & 14 & \multirow[t]{9}{*}{23} & 1 & 6 & 144 & 3312 \\
\hline & 9 & 4 & 4 & 14 & & 1 & 6 & 141 & 6486 \\
\hline \multirow[t]{9}{*}{11} & 1 & 3 & 34 & 1122 & & 1 & 6 & 150 & 1725 \\
\hline & 1 & 3 & 36 & 396 & & 5 & 7 & 42 & 138 \\
\hline & 1 & 3 & 42 & 154 & & 9 & 8 & 24 & 138 \\
\hline & 1 & 3 & 44 & 132 & & 13 & 9 & 16 & 3312 \\
\hline & 1 & 3 & 66 & 66 & & 13 & 9 & 18 & 138 \\
\hline & 5 & 4 & 9 & 396 & & 17 & 10 & 15 & 138 \\
\hline & 5 & 4 & 11 & 44 & & 25 & 12 & 12 & 138 \\
\hline & 5 & 4 & 12 & 33 & \multirow[t]{6}{*}{29} & 3 & 8 & 80 & 2320 \\
\hline & 13 & 6 & 6 & 33 & & 3 & 8 & 87 & 696 \\
\hline \multirow[t]{4}{*}{13} & 3 & 4 & 18 & 468 & & 3 & 8 & 88 & 638 \\
\hline & 3 & 4 & 20 & 130 & & 3 & 8 & 116 & 232 \\
\hline & 3 & 4 & 26 & 52 & & 11 & 10 & 29 & 290 \\
\hline & 7 & 5 & 10 & 130 & & 15 & 11 & 22 & 638 \\
\hline \multirow[t]{4}{*}{17} & 3 & 5 & 30 & 510 & \multirow[t]{4}{*}{41} & 3 & 11 & 154 & 6314 \\
\hline & 3 & 5 & 34 & 170 & & 7 & 12 & 72 & 2952 \\
\hline & 7 & 6 & 15 & 510 & & & & & \\
\hline & 7 & 6 & 17 & 100 & & & & & \\
\hline
\end{tabular}

Table 3: $z / y$ in relation to $p$ and $x$ 


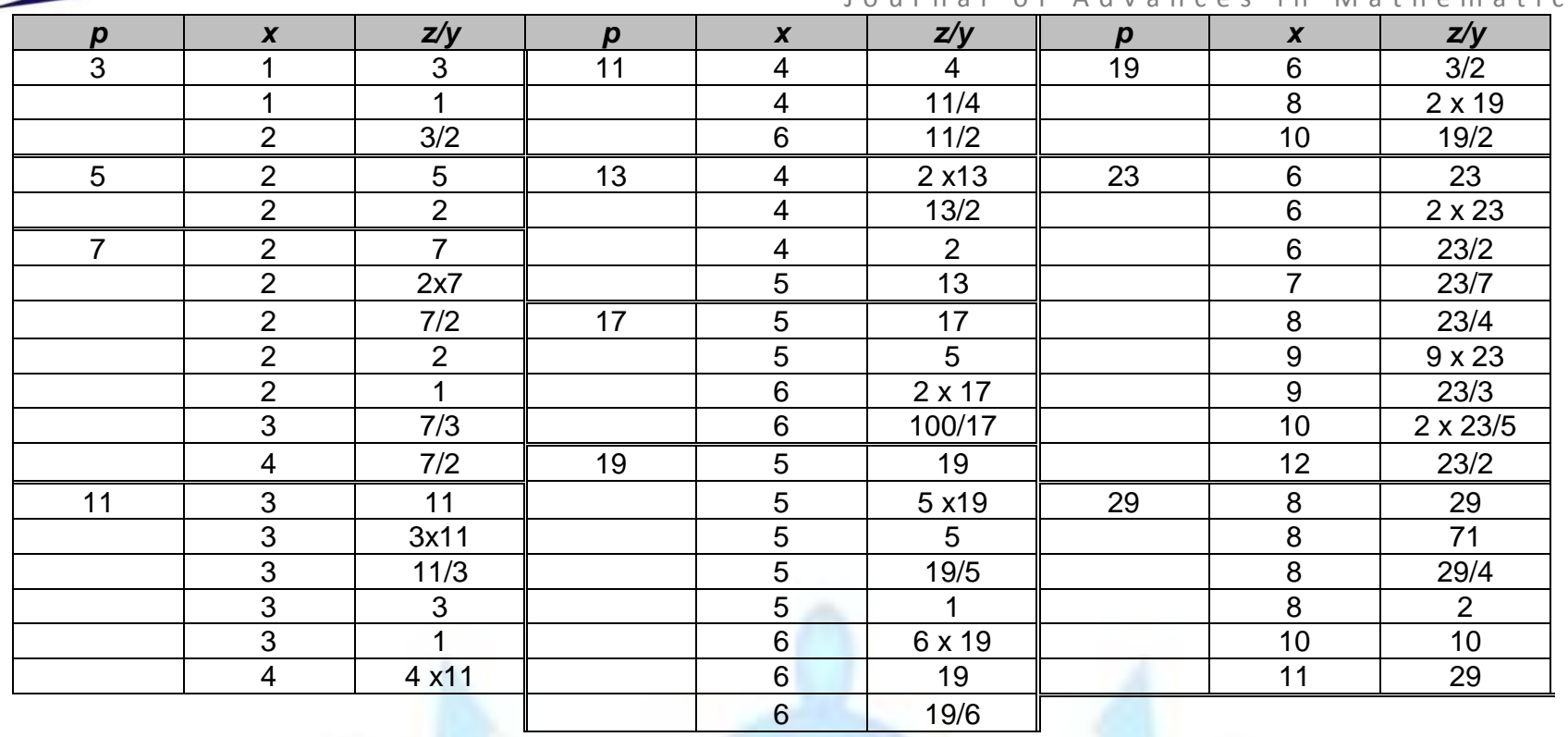

Since odd integers in classes $\overline{1}_{4}$ and $\overline{3}_{4}$ extend to infinity, the parallel lines in Figure 1 below will be intersected by horizontal (parallel to the $x$-axis) lines which represent the primes. While these intersections can yield non-integer $x$, there will be some integer intersections. Thus all primes will have one or more sets of $(x, y, z)$ triples, as will composites since they are products of primes. That is

$$
\frac{4}{p_{1} p_{2}}=\left(\frac{1}{p_{2}}\right)\left(\frac{1}{x}+\frac{1}{y}+\frac{1}{z}\right)
$$

and so on. For example, for $n=6(=2 x 3),(x, y, z)=(2,8,24)$ and for $n=3,(x, y, z)=(1,4,12)$.

To calculate $y$ and $z$ from $B$ it is useful to note that $z / y=p$ or $k p$ (Table 3). Since $B$ is known from the pattern in Table 1, that is, $p \in \overline{1}_{4}$ has $3,7,11, \ldots$, and $p \in \overline{3}_{4}$ has $1,5,9, \ldots$ for $p B$ which corresponds to a particular $x$. Thus when $z / y=p$,

$$
\begin{aligned}
B & =\frac{x\left(\frac{z}{y}+1\right)}{z} \\
& =\frac{x(p+1)}{z}
\end{aligned}
$$

so that

$$
z=\frac{x(p+1)}{B}
$$

and

$$
y=\frac{z}{p} .
$$

For instance, for $p=11, x=3$, and $p B=1$, so that $z=3(12) \times 11 / 1=396$, and $y=396 / 11=36$. Hence, $(x, y, z)=(3,36,96)$ (Table 2). Some examples of larger primes are displayed in Table 4. Notice that when $p \in \overline{1}_{4}, p B \varepsilon\{3,7,11,15, \ldots\}[25$ : A004767] and when $p \in \overline{3}_{4}, p B \varepsilon\{1,5,9,13, \ldots\}$ [25: A016813]. A modification of Table I and parts of Table 4 appears below, followed by a graphical representation which emphasises the consistency of the structures.

Table 4: $(x, y, z)$ for some prime examples [selected at random] 


\begin{tabular}{|c|c|c|c|c|c|c|}
\hline$p$ & $Z_{4}$ & $k$ & $p B$ & $x$ & $z=x(k p+1) / B$ & $y=z / k p$ \\
\hline 101 & $\overline{1}_{4}$ & 1 & 3 & 26 & $26 \times 34 \times 101=89284$ & $26 \times 34=884 ?$ \\
\hline 127 & $\overline{3}_{4}$ & 1 & 1 & 32 & $32 \times 128 \times 127=520192$ & $32 \times 128=4096$ \\
\hline 1307 & $\overline{3}_{4}$ & 1 & 1 & 327 & $327 \times 1308 \times 1307=559024812$ & $327 \times 1308=427716$ \\
\hline 2819 & $\overline{\overline{3}}_{4}$ & 1 & 1 & 705 & $705 \times 2820 \times 2819=5604453900$ & $705 \times 2820=1988100$ \\
\hline 3433 & $\overline{1}_{4}$ & 2 & 7 & 860 & $860 \times 981 \times 3433=2896284780$ & $430 \times 981=421830$ \\
\hline 7121 & $\overline{1}_{4}$ & 1 & 3 & 1781 & $1781 \times 2374 \times 7121=30108257374$ & $1781 \times 2374=4228094$ \\
\hline 22349 & $\overline{1}_{4}$ & 1 & 3 & 5588 & $5588 \times 7450 \times 22349=930402279400$ & $5588 \times 7450=91630600$ \\
\hline 86287 & $\overline{3}_{4}$ & 1 & 1 & 21572 & 21572 x $86288 \times 86287=160615030455232$ & $21572 \times 86288=1861404736$ \\
\hline 99787 & $\overline{3}_{4}$ & 1 & 1 & 24947 & $24947 \times 99788 \times 99787=248410879006732$ & $24947 \times 99788=2489411236$ \\
\hline 530443 & $\overline{3}_{4}$ & 1 & 1 & 132611 & $132611 \times 530444 \times 530443=3.73127977 \mathrm{E} 16$ & $132611 \times 530444=70342709284$ \\
\hline 533993 & $\overline{1}_{4}$ & 2 & 7 & 133499 & $133499 \times 152581 \times 533993=1.08771228 E 16$ & $133499 \times 152581=20369410919$ \\
\hline
\end{tabular}

Table 5: Reconfiguration of Tables 1 and 4

\begin{tabular}{|c|c|c||c|c|c||c|c|c||c|c|c||c|c|c||c|c|c|}
\hline $\boldsymbol{p B}$ & $\boldsymbol{p}$ & $\boldsymbol{x}$ & $\boldsymbol{p} \boldsymbol{B}$ & $\boldsymbol{p}$ & $\boldsymbol{X}$ & $\boldsymbol{p B}$ & $\boldsymbol{p}$ & $\boldsymbol{x}$ & $\boldsymbol{p B}$ & $\boldsymbol{p}$ & $\boldsymbol{x}$ & $\boldsymbol{p B}$ & $\boldsymbol{p}$ & $\boldsymbol{x}$ & $\boldsymbol{p B}$ & $\boldsymbol{p}$ & $\boldsymbol{X}$ \\
\hline 1 & 3 & 1 & 3 & 5 & 2 & 5 & 3 & 2 & 7 & 5 & 3 & 9 & 7 & 4 & 15 & 29 & 11 \\
\hline 1 & 7 & 2 & 3 & 13 & 4 & 5 & 7 & 3 & 7 & 13 & 5 & 9 & 23 & 8 & 17 & 23 & 10 \\
\hline 1 & 11 & 3 & 3 & 17 & 5 & 5 & 11 & 4 & 7 & 17 & 6 & 13 & 11 & 6 & 21 & 19 & 10 \\
\hline 1 & 19 & 5 & 3 & 29 & 8 & 5 & 19 & 6 & 7 & 41 & 12 & 13 & 19 & 8 & 25 & 23 & 12 \\
\hline 1 & 23 & 6 & 3 & 41 & 11 & 5 & 23 & 7 & & & & 13 & 23 & 9 & & & \\
\hline
\end{tabular}

A further refinement of the work in this section would be to investigate finite sums of reciprocals of distinct $n^{\text {th }}$ primes [13], and we look at some other types of finite sums in the next section.

\section{HARMONIC NUMBERS}

Harmonic numbers are sums of the reciprocals of the natural numbers. More precisely, the $n^{\text {th }}$ Harmonic number is the sum of the reciprocals of the first $n$ natural numbers:

$$
H_{n}=\sum_{k=1}^{n} \frac{1}{k} .
$$

For example, $H_{3}=1+\frac{1}{2}+\frac{1}{3}$, so that the components are unit fractions. Moreover, it can be seen that $H_{n}$ is $n$ times the inverse of the harmonic mean of these natural numbers. More generally, the harmonic mean of $n$ numbers $x_{1}, x_{2}, \ldots, x_{n}$ is given by

$$
\begin{aligned}
H\left(x_{1}, x_{2}, \ldots, x_{n}\right) & =\frac{n}{\frac{1}{x_{1}}+\frac{1}{x_{2}}+\ldots+\frac{1}{x_{n}}} \\
& =\frac{\left(G\left(x_{1}, x_{2}, \ldots, x_{n}\right)\right)^{n}}{A\left(x_{2} x_{3} \ldots x_{n}, x_{1} x_{3} \ldots x_{n}, \ldots, x_{1} x_{2} \ldots x_{n-1}\right)} \\
& =\frac{(G(v(n, 1,1)))^{n}}{A(n, 1, n-1)}
\end{aligned}
$$

where $G(\bullet)$ and $A(\bullet)$ represent the geometric and arithmetic means respectively of their arguments which are expressed in terms of symmetric vectors $v(r, t, m)$. Other examples of these vectors include

$$
v(3,1,2)=\left(x_{2} x_{3}, x_{3} x_{1}, x_{1} x_{2}\right)
$$

and

$$
v(n, t, 1)=\left(x_{1}^{t}, x_{2}^{t}, \ldots, x_{n}^{t}\right)
$$

They are related to the symmetric function of $r$ different $x_{i}$ to the power $t$ taken $m$ at a time 


$$
s(r, t, m)=\sum_{j=j(m)} v(r, t, j)
$$

which opens up a whole world of enumeration mathematics [18].

Generalized harmonic numbers of order $r$ can be represented by $H_{n}^{(r)}$ (though this symbol has also been used for hyperharmonic numbers [7]:

$$
H_{n}^{(r)}=\sum_{k=1}^{n} \frac{1}{k^{r}}
$$

so that $H_{n}^{(1)}$ is an ordinary harmonic number and $H_{n}^{(0)}=n$. They satisfy the recurrence relations

$$
H_{n}^{(r)}=H_{n-1}^{(r)}+\frac{1}{n^{r}}
$$

so that

$$
H_{n}^{(0)}=H_{n-1}^{(0)}+\frac{1}{n^{0}}-(n-1)+1=n,
$$

and

$$
H_{n}^{(r)}=\frac{1}{1 \times n} H_{n}^{(r-1)}+\frac{1}{n(n-1)} H_{n-1}^{(r-1)}+\frac{1}{(n-1)(n-2)} H_{n-2}^{(r-1)}+\ldots+\frac{1}{2 \times 1} H_{1}^{(r-1)}
$$

Other properties, such as generating functions, can be readily developed. Consider

the Riemann zeta function.

$$
\lim _{n \rightarrow \infty} H_{n}^{(r)}=\zeta(r)
$$

\section{RECURRENCE RELATIONS}

The harmonic, geometric and arithmetic means all satisfy first order recurrence relations:

$$
G_{n}=r G_{n-1}, A_{n}=A_{n-1}+d
$$

the first being homogeneous (with common ratio $r$ ) and the second non-homogeneous (with common difference $d$ ). The generalized harmonic numbers have two recurrence relations, one of each kind: (3.1) a non-homogeneous recurrence relation for $n$, and (3.2) a homogeneous recurrence relation for $r$.

Fibonacci's Liber Abaci, mentioned in Section 2, effectively introduced the Fibonacci numbers. These can be defined by the second order homogeneous recurrence relation

$$
F_{n}=F_{n-1}+F_{n-2}, n>2
$$

with initial conditions $F_{1}=F_{2}=1$. The general term of the sequence $\left\{F_{n}\right\}$ of Fibonacci numbers is given by the formula

$$
F_{n}=\frac{\varphi^{n}+\left(\frac{1}{\varphi}\right)^{n}}{\sqrt{5}}
$$

where $\varphi=\frac{1}{2}(1+\sqrt{5})$ is the golden ratio. These have many well-known properties which can be explored further by considering the generalized golden ratio, $\frac{1}{2}(1+\sqrt{a})$ and applying this to second order homogeneous linear recurrence relations to get the patterns in Table 6 where $a \equiv 1(\bmod 4)$ and $b=\lfloor a / 4\rfloor$ in which $\lfloor\bullet\rfloor$ is the floor function.

Table 6: Generalized Fibonacci numbers 


\begin{tabular}{|c|c||c|c|c|c|c|c|c|c|c|}
\hline $\boldsymbol{a}$ & $\boldsymbol{b}$ & \multicolumn{6}{c|}{$u_{n}=u_{n-1}+b u_{n-2}, n>2, u_{1}=u_{2}=1$} & Sloane [25] \\
\hline 5 & 1 & 1 & 1 & 2 & 3 & 5 & 8 & 13 & $\ldots$ & $\mathrm{A} 000045$ \\
\hline 9 & 2 & 1 & 1 & 3 & 5 & 11 & 21 & 43 & $\cdots$ & $\mathrm{A} 001045$ \\
\hline 13 & 3 & 1 & 1 & 4 & 7 & 19 & 40 & 97 & $\cdots$ & $\mathrm{A} 006130$ \\
\hline 17 & 4 & 1 & 1 & 5 & 9 & 29 & 65 & 181 & $\cdots$ & $\mathrm{A} 006131$ \\
\hline 21 & 5 & 1 & 1 & 6 & 11 & 41 & 96 & 301 & $\cdots$ & $\mathrm{A} 015440$ \\
\hline 25 & 6 & 1 & 1 & 7 & 13 & 55 & 133 & 463 & $\cdots$ & $\mathrm{A} 015441$ \\
\hline 29 & 7 & 1 & 1 & 8 & 15 & 71 & 176 & 673 & $\cdots$ & $\mathrm{A} 015442$ \\
\hline
\end{tabular}

The patterns across rows, down columns (and diagonals) are worth further investigation in the context of partial recurrence relations with analogies from partial difference equations to partial differential equations. We can also connect the ordinary Fibonacci numbers with unit fractions in continued fractions. As an illustration consider the continued fraction expansion of $\varphi$. Although it is an algebraic irrational number we can specify its continued fraction expansion accurately and precisely. It is an example of the fact that the continued fraction expansion of an irrational number is unique. We start with

$$
\varphi=1+\frac{1}{\varphi}
$$

which we continue

and then

$$
\varphi=1+\frac{1}{1+\frac{1}{\varphi}}
$$

$$
\varphi=1+\frac{1}{1+\frac{1}{1+\frac{1}{\varphi}}}
$$

and so on. We express this in the simple form

$$
\varphi=[1 ; \mathrm{i}]
$$

Likewise the continued fraction expansion of the surd $\sqrt{2}$ can be expressed as

$$
\sqrt{2}=[1 ; \dot{2}]
$$

However, the continued fraction expansions of the transcendental irrational numbers are not so neat; for instance [25],

$$
\pi=[3 ; 7,15,1,292,1,1,1,2,1,3,1,14,2,1,1,2,2,2, \ldots] .
$$

Continued fractions can be used in integer structures for rational approximations of real numbers and Diophantine equations with second order linear recurrence relations $[4,19,20]$ as can their multidimensional generalizations with arbitrary order linear recurrence relations [22]. These computational exercises lead quite naturally into topics in the philosophy of mathematics [5] as foreshadowed in the Introduction to this note.

\section{REFERENCES}

[1] Ahmadi, M.H., M.N. Bleicher. 1998. On the conjectures of Erdös and Straus, and Sierpiński on Egyptian fractions. International Journal of Mathematical and Statistical Sciences. 7 (2): 165-185.

[2] Bailey, D.H., J.M. Borwein, N.J. Calkin, R. Girgensohn, D.R. Luke, V.H. Moll. 2007. Experimental Mathematics in Action. Wellesley, MA: AK Peters.

[3] Bernstein, Leon. 1962. Zur Lösung der diophantischen Gleichung $m / n=1 / x+1 / y+1 / z$, insbesondere im Fall $m=4$. Journal für die Reine und Angewandte Mathematik. 211: 1-10.

[4] Bernstein, Leon. 1967. The generalized Pellian equation. Transactions of the American Mathematical Society. 127 
(1): 76-89.

[5] Burgess, John P. 2015. Rigor and Structure. Oxford: Oxford University Press.

[6] Castellanos, D. 1986. Rapidly converging expansions with Fibonacci coefficients. The Fibonacci Quarterly. 24 (1): 70-82.

[7] Conway, John H., Richard K Guy. 1995. The Book of Numbers. New York: Copernicus/Springer, p.258.

[8] Crothers, John N., Anthony G. Shannon. 2016. Quantics: Diophantine Congruences in Special Fibonacci Lattices. Ahmedabad: Sara Books.

[9] Eves, Howard. 1990. An Introduction to the History of Mathematics. $6^{\text {th }}$ Edition. Philadelphia, PA: Saunders.

[10] Franklin, James. 2015. The Science of Conjecture. Baltimore, MD: Johns Hopkins University Press.

[11] Freitag, Herta T, George M. Phillips. 1999. Sylvester's algorithm and Fibonacci numbers. In Fredric T Howard (ed), Applications of Fibonacci Numbers, Volume 8. Dordrecht: Kluwer, pp.155-163.

[12] Gillings, Richard J. 1964. Mathematics in the Time of the Pharaohs. New York: Dover.

[13] Graham, R.L. 1964. On finite sums of reciprocals of distinct $n^{\text {th }}$ primes. Pacific Journal of Mathematics. 14 (1): $85-92$.

[14] Hagedorn, Thomas R. 2000. A proof of a conjecture on Egyptian fractions. American Mathematical Monthly. 107 (1): 62-63.

[15] Hoffman, Paul. 1998. The Man Who Loved Only Numbers: The Story of Paul Erdös and the Search for Mathematical Truth. New York: Hyperion, pp.153-157.

[16] Leyendekkers, J.V., A.G. Shannon. 2006. Using Integer Structure to Solve Diophantine Equations. Notes on Number Theory and Discrete Mathematics. 12 (3): 10-19.

[17] Leyendekkers, J.V., A.G. Shannon, J. Rybak. 2007. Pattern Recognition: Modular Rings and Integer Structure. North Sydney: RafflesKvB Monograph No.9.

[18] Macmahon, Percy A. 1915. Combinatory Analysis. Volume 1. Cambridge University Press.

[19] Mordell, Louis J. 1967. Diophantine Equations. New York: Academic Press, pp.287-290.

[20] Shannon, A.G. 1976. Pellian equations and continued fractions. New Zealand Mathematics Magazine. 13(3): 113115.

[21] Shannon, A.G. 1991. Shrewd guessing in problem-solving. International Journal of Mathematical Education in Science and Technology. 22 (1):144-147.

[22] Shannon, A.G., Leon Bernstein. 1973. The Jacobi-Perron Algorithm and the algebra of recursive sequences. Bulletin of the Australian Mathematical Society. 8 (): 261-277.

[23] Sierpiński, Wacław. 1956. Sur les decompositions de nombres rationneles en fractions primaries. Mathesis. 65 : 16 32.

[24] Sigler, L.E. 2002. Fibonacci's Liber Abaci: A Translation into Modern English of Leonardo Pisano's Book of Calculation. New York: Springer, Ch.7.

[25] Sloane, N.J.A. 1964+. https://oeis.org/.

[26] Webb, William A. 1970. On $4 / n=1 / x+1 / y+1 / z$. Proceedings of the American Mathematical Society. 25 (3): $578-$ 584.

[27] White, Sonia L.J. 2014. Transitioning from Vocational Education and Training to University: Strengthening Information Literacy through Collaboration. Adelaide: National Centre for Vocational Education Research. 


\section{Authors' biographies with Photos}

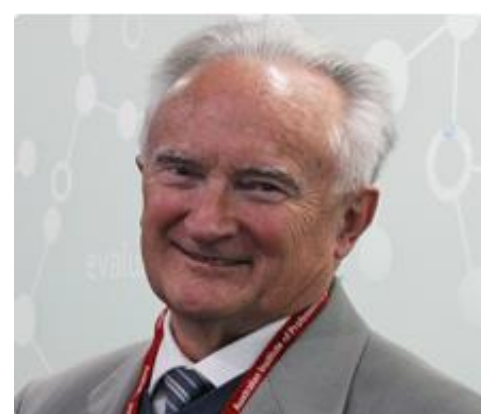

Professor A. G. (Tony) Shannon AM is an Emeritus Professor of the University of Technology, Sydney, where he was Foundation Dean of the Graduate Research School. He holds the degrees of PhD, EdD and DSc. He is co-author of numerous books and articles in medicine, mathematics and education. His research interests are in the philosophy of education, number theory, and epidemiology, particularly through the application of generalized nets and intuitionistic fuzzy logic. Professor Shannon is a Fellow of several professional societies. He is presently part-time Registrar of Campion College, a liberal arts degree granting institution in Sydney, and part-time Academic Dean of the Australian Insitute of Music. In 1987 in the Queen's Birthday Honours he was appointed a Member of the Order of Australia (AM) for services to education.

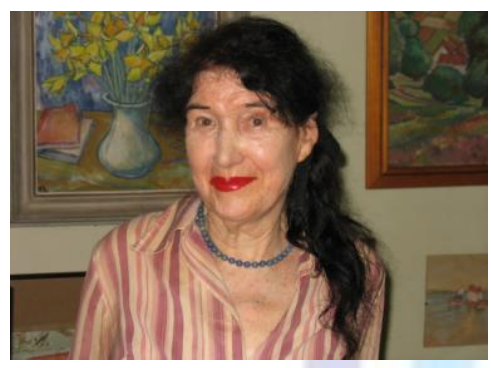

Dr Jean Leyendekkers was awarded a Doctor of Science (D.Sc) degree by the University of Sydney for her published work on Solution Theory. Since officially retiring from the Faculty of Science there Jean has written papers on Number Theory and now has ninety published. A few of the earlier papers were written with Janet Rybak but most have since been co-authored with Professor Tony Shannon. The emphasis has been on the Integer Structure influence in Number Theory, including the applications in conjectures with experimental mathematics. Jean has been active for 30 years in community work on urban planning and in particular on the regeneration of bushland. Jean enjoys classical music, mysteries and loves cats, dogs, possums and all other animals and birds.

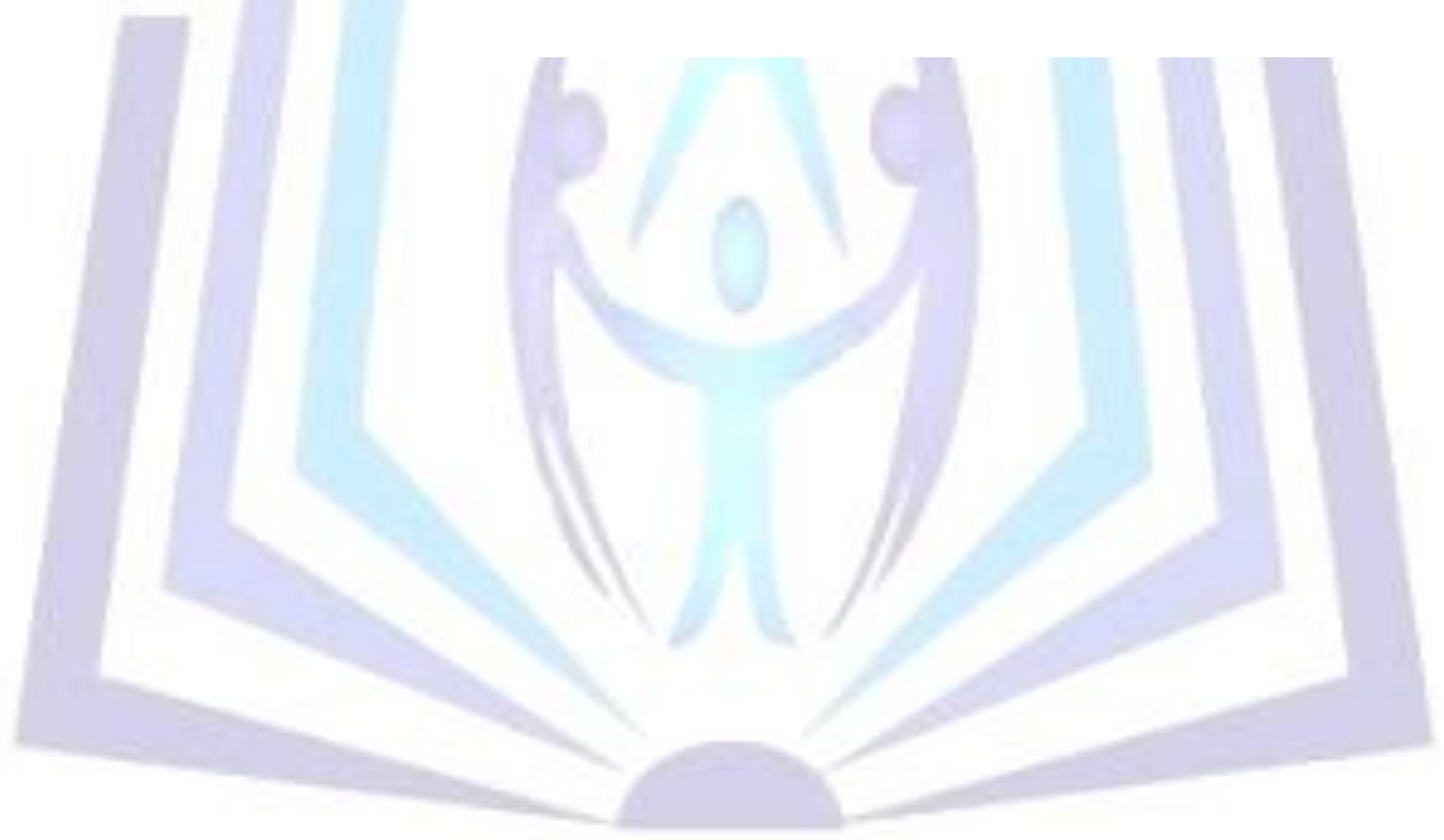

